\title{
Including the Sheath Rinse to Improve Cellular Yield in Biliary Brushing Cytology
}

\author{
Glenda F. Amog-Jones ${ }^{1}$, Subhash Chandra ${ }^{2}$, Chris Jensen ${ }^{3}$ and Frederick C. Johlin ${ }^{2}$ \\ ${ }^{1}$ Department of Pathology, Phoenix VA Health Care System, Phoenix, AZ, ${ }^{2}$ Division of Gastroenterology and Hepatology, ${ }^{3}$ Department of \\ Pathology, University of lowa Hospitals and Clinics, lowa City, IA, USA
}

The incidence of intrahepatic cholangiocarcinoma has been increasing in the last few decades. ${ }^{1}$ Interestingly over this period, the incidence of extrahepatic cholangiocarcinoma remained stable. ${ }^{1}$ However, the proportion of patients with early stage, small or localized tumors remained the same. ${ }^{2}$ This reflects a true increase in the incidence without a corresponding significant gain in our ability to detect cholangiocarcinoma at an earlier stage. As a result, the cholangiocarcinoma related mortality has also increased significantly.

Primary sclerosing cholangitis (PSC) is a major risk factor for cholangiocarcinoma in the Western population. Although identification of cholangiocarcinoma at an earlier stage has significantly better outcomes, our ability to detect these early cancers is limited. Tumor markers (CA 19-9 and CEA) may have value for screening in some settings, but effectiveness for detecting small early stage tumors is questionable and magnetic resonance cholangiopancreatography is used in some centers without robust evidence to support its utility. Currently endoscopic retrograde cholangiopancreatography (ERCP) with brush cytology or biopsy is not recommended as screening modality in PSC due to its low sensitivity.

However, ERCP with brush cytology is recommended for

Received: May 25, 2017 Revised: August 25, 2017

Accepted: August 26, 2017

Correspondence: Frederick C. Johlin

Division of Gastroenterology and Hepatology, University of Iowa Hospitals and Clinics, 200 Hawkins drive, 4553D JCP, Iowa City, IA 52242, USA

Tel: +1-319-356-4030, Fax: +1-319-535-6399, E-mail: fredjohlin@msn.com

cc This is an Open Access article distributed under the terms of the Creative Commons Attribution Non-Commercial License (http://creativecommons.org/ licenses/by-nc/3.0) which permits unrestricted non-commercial use, distribution, and reproduction in any medium, provided the original work is properly cited. sampling biliary and pancreatic strictures, but sensitivity continues to be low. False negative results are usually attributed to limitation in cell acquisition and corresponding low cellularity. Techniques have been attempted to improve cell acquisition, including use of intraductal biopsies, alone or in combination with brush cytology and use of large bristle versus small bristle brushes have been examined. ${ }^{3,4}$ Diagnostic accuracy of biliary brushing at our center has been higher than what is reported in the literature. ${ }^{5}$ We theorize that it might be due to the fact that we submit both the brush (cut from the neck) and sheath rinse which, to our knowledge, is not a common practice. The underlying hypothesis for this technique is that during the brushing, cells may be dislodged from the brush into the protective sheath. The sheath scrapes the duct wall in the area of stricture potentially further dislodging cellular material into the sheath. The aim of this study was to determine impact of incorporating sheath rinsing on cellular yield of biliary brush cytology.

This prospective study was performed at am US tertiary care center. The study protocol was approved by the institutional review board. Consecutive patients who underwent ERCP over a one month period for biliary strictures were enrolled. The procedure was performed by an advanced endoscopist with more than 20 years of experience performing ERCP. The specimens were obtained during the ERCP by brushing the common bile duct, common hepatic duct, and right and left hepatic ducts. A ConMed ${ }^{\circledR}$ sheathed colonoscopy brush was used. The brush diameter is $3.0 \mathrm{~mm}$ and the sheath outer diameter is $2.1 \mathrm{~mm}$. The brush and the sheath were advanced above the stricture using a monorail technique. The lead edge of the sheath and the brush were advanced and withdrawn through the stricture for a total 
Table 1. Assessment of Cellularity between Thin Layer and Cell Block Preparations of Brushing and Sheath Rinsing

\begin{tabular}{|c|c|c|c|c|c|c|}
\hline \multirow{2}{*}{ Specimen } & \multicolumn{3}{|c|}{ Brushing } & \multicolumn{3}{|c|}{ Sheath } \\
\hline & Thin layer & Cell block & Overall & Thin layer & Cell block & Overall \\
\hline 1 & 1 & 2 & 2 & 0 & 2 & 2 \\
\hline 2 & 1 & 2 & 2 & 0 & 2 & 2 \\
\hline 3 & 0 & 2 & 2 & 1 & 2 & 2 \\
\hline 4 & 1 & 1 & 1 & 1 & 2 & 2 \\
\hline 5 & 3 & 2 & 3 & 3 & 2 & 3 \\
\hline 6 & 2 & 1 & 2 & 2 & 2 & 2 \\
\hline 7 & 2 & 1 & 2 & 1 & 2 & 2 \\
\hline 8 & 2 & 1 & 2 & 3 & $\mathrm{NP}$ & 3 \\
\hline 9 & 1 & 0 & 1 & 1 & 1 & 1 \\
\hline 10 & 2 & 1 & 2 & 2 & 1 & 2 \\
\hline 11 & 1 & $\mathrm{NP}$ & 1 & 3 & 1 & 3 \\
\hline 12 & 0 & $\mathrm{NP}$ & 0 & 0 & 1 & 1 \\
\hline 13 & 1 & 1 & 1 & 0 & 1 & 1 \\
\hline
\end{tabular}

NP, not prepared.

of 15 in and out movements. Paired brush and sheath rinse were done for the same site and submitted separately. The brush was cut off the wire into the fixative. The sheath was rinsed into a separate container using a $30 \mathrm{cc}$ syringe to aspirate and flush into and out of the fixative. Our standard practice is to submit brush and rinse together as a single sample from each site. All samples were submitted in 50\% methanol. Thin-layer (Autocyte; TriPath Inc., Burlington, NC, USA) and cell block preparations were prepared using our standard technique.

The cellularity of the specimens was assessed by a single cytopathologist who was blinded to the method of specimen acquisition. Cellularity was graded on a scale described by Silverman et al. as "0" unsatisfactory (no clusters of cells or sheets), "1" low (few single cells or small sheets), "2" moderate (few to many small clusters and few large sheets) and "3" high (many small clusters and large sheets). The cellularity scores of corresponding paired preparations were combined to determine overall cellularity. Observations are reported as proportions.

Twenty-six (13 paired) biliary samplings were analyzed. The cellularity from individual specimen on thin layer and cell block preparations are presented in Table 1. Cell blocks could not be prepared due to lack of visible material from 2 brush specimens and one sheath rinse specimen. In these specimens, the thin layer cellularity was rated high in the sheath rinse specimen and unsatisfactory in one and low in another specimen obtained from the brush.

The overall cellularity was ranked moderate or high in 8 out of $13(62 \%)$ specimens obtained from the brush and 10 out of $13(77 \%)$ specimen obtained from the sheath rinse. One brush specimen had unsatisfactory cellularity whereas every sheath specimen had some degree cellularity. In all the specimens, sheath rinse had equal or better cellularity compared to brush. Four of 13 (30\%) specimens using sheath rinse showed better yield than brushing specimens. The combination of the two techniques showed moderate to high cellularity in 10 of 13 (77\%) specimens and low cellularity in 3 specimens.

Cellular yield in brush cytology from biliary stricture is extremely important as inadequate cellularity is considered one the main reason of low sensitivity for biliary malignancies. In this pilot study, we demonstrate a potential solution. We have demonstrated that the cellular yield of brush cytology is higher when the sheath rinse is combined with the brush instead of submitting the brush alone. In addition to capturing detached cells from the brush, moving both the sheath across the stricture also may dislodge deeper cells, as the sheath scraps off cells from below the mucosa which has been abraded by the brush. This improves the sensitivity as the cellular portion of biliary lesions is often submucosal.

Biliary tract brushing cytology is the modality of choice in evaluating stricture as it allows cell acquisition for diagnosis. Several modalities are described in literature in an effort to increase the sensitivity, but none of them are widely adopted. Combining cytology with digital image analysis or Fluorescence in situ hybridization of bile duct brushing is well studied. These techniques look for aneuploidy which is 
a limitation as not all the pancreatobiliary malignancies have aneuploidy. ${ }^{6}$ Inflammatory stricture in PSC often have chromosomal abnormalities. In these cases, serial examinations are needed to improve specificity. ${ }^{6,7}$

The combination of stricture balloon dilatation, endoscopic needle aspiration, and subsequent biliary brushing has been reported to increase sensitivity, but the procedure has not been widely adopted as it is cumbersome, lengthy and adds additional cost from balloon and needle. ${ }^{8}$

Brush cytology has better sensitivity compared bile aspirate in biliary malignancies. Abrasive bristles dislodge cells from ductal lesions and improve the cellularity. Bile aspirate after brush cytology has been reported to improve cellular yield., ${ }^{9,10}$ This likely reflects that cellular material from the bristles falls off into the lumen or into the brush sheath. As such, our observations are similar to the increase in yield demonstrated with post brushing bile aspirate likely reflecting cellular material drawn into the sheath from the bile duct lumen.

Of note, our technique does not require additional instruments or significant time to generate additional cellular yield. It simply requires the endoscopist to pass the sheath into the stricture and move the brush and the sheath in and out of the stricture for at least 15 cycles. The brush and sheath rinsing can then be submitted together for cytologic evaluation. Communication with the cytology lab may be necessary to insure that the fluid is processed in conjunction with the brush as is likely common practice in most labs.

We report a simple modification in technique to improve cellular yield of brush cytology for biliary lesions. Moving the sheath and brush across the stricture for at least 15 times and sending the sheath rinse along with the brush, improves cellular yield of brush cytology for biliary stricture. This technique does not require additional instruments or significant efforts to improve cellular yield. Based on the simplicity and no additional cost, it has potential for widespread adoption.

\section{Conflicts of Interest}

The authors have no financial conflicts of interest.

\section{Author Contributions}

Conceptualization: Chris Jensen, Frederick C. Johlin

Data curation: Glenda F. Amog-Jones, Subhash Chandra

Formal analysis: GFAJ, SC, FCJ

Investigation: GFAJ, FCJ

Methodology: FCJ

Project administration: GFAJ, FCJ

Resources: CJ

Supervision: CJ, FCJ

Validation: FCJ

Visualization: FCJ

Writing-original draft: GFAJ

Writing-review\&editing: SC, CJ, FCJ

\section{REFERENCES}

1. Saha SK, Zhu AX, Fuchs CS, Brooks GA. Forty-year trends in cholangiocarcinoma incidence in the U.S.: intrahepatic disease on the rise. Oncologist 2016;21:594-599.

2. Shaib YH, Davila JA, McGlynn K, El-Serag HB. Rising incidence of intrahepatic cholangiocarcinoma in the United States: a true increase? J Hepatol 2004;40:472-477.

3. Silverman WB, Jensen CS, Crook TW, Henke A. Bile duct brushings in a pig model: examination of intraobserver variability and variability in specimen quality obtained in sequential animals and between two different brushes. Int J Gastrointest Cancer 2002;32:31-34.

4. Navaneethan U, Njei B, Lourdusamy V, Konjeti R, Vargo JJ, Parsi MA. Comparative effectiveness of biliary brush cytology and intraductal biopsy for detection of malignant biliary strictures: a systematic review and meta-analysis. Gastrointest Endosc 2015;81:168-176.

5. Cohen MB, Wittchow RJ, Johlin FC, Bottles K, Raab SS. Brush cytology of the extrahepatic biliary tract: comparison of cytologic features of adenocarcinoma and benign biliary strictures. Mod Pathol 1995;8:498-502.

6. Bangarulingam SY, Bjornsson E, Enders F, et al. Long-term outcomes of positive fluorescence in situ hybridization tests in primary sclerosing cholangitis. Hepatology 2010;51:174-180.

7. Barr Fritcher EG, Kipp BR, Voss JS, et al. Primary sclerosing cholangitis patients with serial polysomy fluorescence in situ hybridization results are at increased risk of cholangiocarcinoma. Am J Gastroenterol 2011;106:2023-2028.

8. Farrell RJ, Jain AK, Brandwein SL, Wang H, Chuttani R, Pleskow DK. The combination of stricture dilation, endoscopic needle aspiration, and biliary brushings significantly improves diagnostic yield from malignant bile duct strictures. Gastrointest Endosc 2001;54:587-594.

9. Curcio G, Traina M, Mocciaro F, et al. Intraductal aspiration: a promising new tissue-sampling technique for the diagnosis of suspected malignant biliary strictures. Gastrointest Endosc 2012;75:798-804.

10. Sugimoto S, Matsubayashi H, Kimura H, et al. Diagnosis of bile duct cancer by bile cytology: usefulness of post-brushing biliary lavage fluid. Endosc Int Open 2015;3:E323-E328. 